\title{
Give me a sign, any sign
}

\section{Peter J Mazzone}

For clinical progress to occur in lung cancer, advances must be made in many inter-related areas. Advances in chemoprevention will be most useful if testing is able to identify those at greatest risk of developing lung cancer. Advances in surgical and ablative therapies will be most useful if testing is able to identify lung cancer at the earliest possible stage. Advances in systemic, targeted and individualised therapies will be most useful if testing is able to predict the nature of a patient's lung cancer and the response to specific treatment choices.

A new test can improve on currently used tests by being more accurate, less invasive, less expensive and/or novel in its intent. To have a clinical impact, the result of the test must affect a decision to the benefit of the patient. The most recently developed tests that have had this sort of impact in lung cancer are positron emission tomographic imaging, ${ }^{1}$ advances in diagnostic bronchoscopy (electromagnetic navigation, ${ }^{2}$ endobronchial ultrasound ${ }^{3}$ ) and perhaps epidermal growth factor receptor analysis. ${ }^{4}$

Progress is occurring on many fronts in lung cancer testing. The clinical impact of imaging advances such as dual energy imaging, ${ }^{5}$ temporal subtraction, ${ }^{6}$ computer-aided detection/diagnosis ${ }^{7}$ and volumetric analysis ${ }^{8}$ of lung nodules is being assessed. Bronchoscopic advances in the use of ultrathin bronchoscopes, ${ }^{9}$ navigation programmes, ${ }^{2}$ ultrasound-guided sampling procedures, ${ }^{3}$ narrow band imaging $^{10}$ and optical coherence tomography ${ }^{11}$ are occurring. We have seen advances in molecular testing through the analysis of tumour and airway tissue genomes ${ }^{12}{ }^{13}$ and proteomes, ${ }^{14}$ as well as blood proteomes, ${ }^{15}$ DNA methylation, ${ }^{16}$ circulating tumour cells, ${ }^{17}$ antibodies to tumourassociated antigens ${ }^{18}$ and microRNA profiles. ${ }^{19}$ Finally, there is hope that analysis of the volatile and non-volatile ${ }^{20}$ components of exhaled breath will provide useful information about our patients.

In this issue of Thorax, progress on the testing of exhaled breath volatile compounds as a diagnostic in lung cancer is presented. Volatile organic compounds

Correspondence to: Dr P J Mazzone, The Cleveland Clinic, 9500 Euclid Avenue, Cleveland, OH 44195, USA; mazzonp@ccf.org
(VOCs) are present in the breath in very low concentrations (parts per billion to parts per trillion volume). They can be inhaled into the lungs and absorbed through the skin from exogenous sources, or can be generated directly from the cellular biochemical processes of the body (eg, lipid peroxidation of fatty acid components of cell membranes). The origin of many endogenous VOCs is not known. Several lines of evidence suggest that the biochemical processes of lung cancer cells differ from those of normal cells. Thus, it is reasonable to expect that the pattern of exhaled volatile compounds in patients with lung cancer would be different from that of individuals without lung cancer.

The concept that a unique pattern of exhaled VOCs exists in those with lung cancer has been studied previously. Some researchers have evaluated gas chromatography/mass spectrometry (GC/MS) systems, while others have investigated the use of non-specific chemical sensing matrix devices for this purpose. There are benefits and downsides to the use of either of these technologies. GC/MS devices are able to identify the specific components of a gas mixture at low concentrations, but they are cumbersome to use and expensive. Chemical sensing matrix devices are easier to use as a point of care test and relatively inexpensive, but they do not identify the actual compounds and may lack enough sensitivity to various volatile compounds to be accurate. Despite these concerns, both techniques have shown promise with accuracies in the $70-85 \%$ range for the identification of lung cancer being reported. The studies have differed in the breath collection methods used, the populations tested and the statistical methods applied to identify the unique patterns as well as to validate the model developed. $^{21}$

Technological advances should lead to progress in breath testing. Chemical sensing devices have been developed that can detect VOCs at lower concentrations than traditional GC/MS in near real-time. In this issue of Thorax, Westhoff et al ${ }^{22}$ describe the use of one such device for the analysis of exhaled breath (see page 744). This device, called an ion mobility spectrometer, is able to detect volatile compounds at a tenfold lower concentration than standard GC/MS devices in approximately one-fifth of the time. It does not, however, identify the specific compounds in the mixture. The authors enrolled subjects with lung cancer and healthy controls. They were able to demonstrate a complete separation of the breath signals between these groups. These results hold promise for this or similar devices to be developed into an accurate diagnostic tool, but should be viewed as pilot information only. The authors did not include control subjects with other medical problems, their control group was younger than the cancer group, most of the cancer subjects had relatively advanced disease and they did not use a separate validation cohort. All of these factors may influence the accuracy of the tested device in future studies.

The field of lung cancer test development is on the verge of some major advances. In the near future we may have testing available that will help us to assess the risk of developing lung cancer, diagnose lung cancer at an earlier stage, improve our ability to predict the course of lung cancer once diagnosed and intelligently individualise treatment decisions. These tests will foster advances in other areas of lung cancer management to the benefit of our patients.

\section{Competing interests: None.}

Thorax 2009;64:737-738. doi:10.1136/thx.2008.107409

\section{REFERENCES}

1. Pepe G, Rossetti C, Sironi S, et al. Patients with known or suspected lung cancer: evaluation of clinical management changes due to $18 \mathrm{~F}$-fluorodeoxyglucose positron emission tomography (18F-FDG PET) study. Nucl Med Commun 2005;26:831-7.

2. Gildea TR, Mazzone PJ, Karnak D, et al. Electromagnetic navigation diagnostic bronchoscopy. A prospective study. Am J Respir Crit Care Med 2006;174:982-9.

3. Wallace MB, Pascual JMS, Raimondo M, et al. Minimally invasive endoscopic staging of suspected lung cancer. JAMA 2008;299:540-6.

4. Eberhard DA, Giaccone G, Johnson BE. Biomarkers of response to epidermal growth factor receptor inhibitors in non-small-cell lung cancer working group: standardization for use in the clinical trial setting. J Clin Oncol 2008;26:983-94.

5. Uemura M, Miyagawa M, Yasuhara Y, et al. Clinical evaluation of pulmonary nodules with dual-exposure dual-energy subtraction chest radiography. Radiat Med 2005;23:391-7

6. Johkoh T, Kozuka T, Tomiyama N, et al. Temporal subtraction for detection of solitary pulmonary nodules on chest radiographs: evaluation of a commercially available computer-aided diagnosis system. Radiology 2002;223:806-11.

7. Das M, Muhlenbruch G, Mahnken AH, et al. Small pulmonary nodules: effect of two computer-aided detection systems on radiologist performance. Radiology 2006;241:564-71.

8. Bolte H, Jahnke T, Schafer FKW, et al. Interobservervariability of lung nodule volumetry considering 
different segmentation algorithms and observer training levels. Eur J Radiol 2007;64:285-95.

9. Shinagawa N, Yamazaki K, Onodera Y, et al. Factors related to diagnostic sensitivity using an ultrathin bronchoscope under CT guidance. Chest 2007; 131:549-53.

10. Vincent BD, Fraig M, Silvestri GA. A pilot study of narrow-band imaging compared to white light bronchoscopy for evaluation of normal airways and premalignant and malignant airways disease. Chest 2007;131:1794-9.

11. Coxson HO, Quiney B, Sin DD, et al. Airway wall thickness assessed using computed tomography and optical coherente tomography. Am J Respir Crit Care Med 2008:177:1201-6.

12. Shedden K, Taylor JMG, Enkemann SA, et al. Gene expression-based survival prediction in lung adenocarcinoma: a multi-site, blinded validation study. Nat Med 2008;14:822-7.
13. Spira A, Beane JE, Shah V, et al. Airway epithelial gene expression in the diagnostic evaluation of smokers with suspect lung cancer. Nat Med 2007:13:361-6.

14. Yanagisawa K, Shyr Y, Xu BJ, et al. Proteomic patterns of tumour subsets in non-small-cell lung cancer. Lancet 2003:362:433-9.

15. Yildiz PB, Shyr Y, Rahman JSM, et al. Diagnostic accuracy of MALDI mass spectrometric análysis of unfractionated serum in lung cancer. J Thorac Oncol 2007;2:893-901

16. Brock MV, Hooker CM, Ota-Machida E, et al. DNA methylation markers and early recurrence in stage I lung cancer. N Engl J Med 2008;358:1118-28.

17. Maheswaran S, Sequist LV, Nagrath S, et al. Detection of mutations in EGFR in circulating lungcancer cells. N Engl J Med 2008;359:1-12.

18. Chapman CJ, Murray A, McElveen JE, et al. Autoantibodies in lung cancer: possibilities for early detection and subsequent cure. Thorax 2008;63:228-33.

19. Chen X, Ba Y, Ma L, et al. Characterization of microRNAs in serum: a novel class of biomarkers for diagnosis of cancer and other diseases. Cell Res 2008;18:997-1006.

20. Carpagnano GE, Foschino-Barbaro MP Spanevello A, et al. 3p microsatellite signature in exhaled breath condensate and tumor tissue of patients with lung cancer. Am J Respir Crit Care Med 2008;177:337-41.

21. Mazzone PJ. Analysis of volatile organic compounds in the exhaled breath for lung cancer diagnosis. J Thorac Oncol 2008;3:774-80.

22. Westhoff M, Litterst $P$, Freitag $L$, et al

Ion mobility spectrometry for the detection of volatile organic compounds in exhaled breath of patients with lung cancer: results of a pilot study. Thorax 2009;64:744-8.

\section{The $\beta_{2}$ receptor and airway hyper-responsiveness: are sensory nerves involved?}

\section{Clive Page}

The use of $\beta_{2}$ agonists for the control of symptoms is central to the treatment of patients with asthma. However, there is controversy surrounding the regular use of this drug class as numerous studies have demonstrated a variety of changes that can be considered unwanted attributes, particularly when these drugs are used regularly as monotherapy. These include increased bronchial hyper-responsiveness (BHR) to inhaled contractile agents $^{1}$ and an increase in the allergeninduced early ${ }^{2}$ and late asthmatic response $^{3}$ following regular treatment with short-acting $\beta_{2}$ agonists (SABAs). Furthermore, a number of studies have suggested that regular treatment with inhaled SABAs and long-acting $\beta_{2}$ agonists (LABAs) by inhalation leads to a loss of bronchoprotection ${ }^{4}{ }^{5}$ and with salmeterol treatment an excess mortality in patients with asthma, ${ }^{6}$ a trend also observed with regular treatment with formoterol. ${ }^{7}$ This has led the Food and Drug Administration (FDA) to post black-box warnings on all medicines containing these LABAs.

Christian Virchow and colleagues from Rostock have provided data (see page 763) on a potential mechanism as to how regular treatment with salmeterol can

Correspondence to: Professor Clive Page, Sackler Institute of Pulmonary Pharmacology, Division of Pharmaceutical Sciences, King's College London, Guy's Campus, London Bridge, London SE1 9RT, UK; clive.page@kcl.ac.uk paradoxically increase $\mathrm{BHR} .^{8}$ Eighteen patients with mild allergic asthma inhaled standard doses of salmeterol xinafoate for 2 weeks, followed by 2 weeks of treatment with the combination of fluticasone and salmeterol xinafoate. There was no overall statistically significant change in BHR for the whole group receiving monotherapy with salmeterol. However, $67 \%$ of the patients showed a modest increase in BHR as measured by a lowered $\mathrm{PC}_{20}$ (provocative concentration of histamine causing a $20 \%$ fall in the forced expiratory volume in $1 \mathrm{~s}$ ) following monotherapy with salmeterol compared with baseline. This contrasted with a statistically significant improvement in BHR following the combination therapy. The levels of brain-derived neurotrophic factor (BDNF) were elevated in both serum and platelets obtained from patients receiving monotherapy with salmeterol, and the changes in BDNF levels correlated with the changes in $\mathrm{PC}_{20}$, although the levels of BDNF decreased significantly and there was no such correlation with changes in $\mathrm{PC}_{20}$ in the patients receiving the combination therapy. The changes in $\mathrm{PC}_{20}$ following monotherapy with inhaled salmeterol did not show a correlation with known $\beta_{2}$ receptor polymorphisms.

A number of other investigators have suggested a role for BDNF in BHR as this is a mediator that is increased in subjects with asthma, both in the lung and in platelets, ${ }^{10}$ and which at least in animal models can induce BHR associated with changes in neuronal activity. ${ }^{11}$ In patients with asthma, the systemic levels of BDNF are also elevated, whilst they correlate with BHR. ${ }^{9}$ Increases in BDNF levels in the lung following allergen challenge of patients with asthma can be reduced by glucocorticosteroids. ${ }^{12}$ Airway sensory nerves have also been implicated in the pathogenesis of BHR induced by a number of stimuli, ${ }^{13}$ including treatment with regular $\beta_{2}$ agonists, ${ }^{14}$ and it is of particular interest that platelet activation has also been observed to play a central role in allergen-induced BHR experimentally, ${ }^{15}{ }^{16}$ supporting the observations of Virchow and colleagues in the present study. Interestingly, salmeterol enhanced the secretion of BDNF from tumour necrosis factor $\alpha(T N F \alpha)$-stimulated human peripheral blood mononuclear cells, whilst BDNF secretion was inhibited by fluticasone.

Clearly it would be of interest to see if salmeterol also caused an increase in BDNF secretion from platelets, thus allowing a clearer link between platelet activation, BDNF and the exacerbation of BHR observed following monotherapy with regular inhaled salmeterol. Whilst the acute benefits of $\beta_{2}$ agonist therapy are well accepted, the worsening of asthma control with chronic $\beta_{2}$ agonist treatment is not as well accepted, with a recent study reinforcing the safety of regular $\beta_{2}$ agonists use. ${ }^{17}$ Nonetheless, a number of mechanisms have been put forward to explain worsening asthma control with regular $\beta_{2}$ agonist treatment, including increased antigen burden, ${ }^{18}$ increased BHR induced by the (+) enantiomer $^{14}$ and loss of bronchoprotection. ${ }^{4}$ Recently, the role of $\beta_{2}$ receptors in asthma has become more complicated with the recognition that $\beta$-blockers, which have traditionally been contraindicated in the treatment of patients with 